Metallomics 2012, 4(7), 679-685

\title{
Comparison between copper and cisplatin transport mediated by human copper transporter 1 (hCTR1)
}

\author{
Xiubo Du, ${ }^{a, b}$ Xinghao Wang, ${ }^{a}$ Hongyan $\mathrm{Li}^{a}$ and Hongzhe Sun*a
}

Copper transporter 1 (CTR1) is a transmembrane protein that imports copper(I) in yeast and mammalian cells. Surprisingly, the protein also mediates the uptake of platinum anticancer drugs, e.g. cisplatin and carboplatin. To study the effects of several metal-binding residues/motifs of hCTR1 on the transport of both $\mathrm{Cu}^{+}$and cisplatin, we have constructed Hela cells that stably express a series of hCTR1 variant proteins including H22-24A, NHA, C189S, CT178, H139R and Y156A, and compared their abilities to regulate the accumulation and cytotoxicity of these metal compounds. Our results demonstrated that the cells expressing the hCTR 1 mutants of histidine-rich motifs in the N-terminus (H22-24A, NHA) resulted in a higher basal copper level in the steady state compared to those expressing wild-type protein. However, the cellular accumulation of both copper and cisplatin in these variants was found on a similar level to that of wild type when incubated with excess amounts of metal compounds $(100 \mu \mathrm{M})$. The cells expressing hCTR1 variants of H139R and Y156A exhibit lower capacities towards accumulation of copper but not cisplatin. Significantly, cells with the C189S variant partially retained the ability of the wild-type hCTR1 protein to accumulate both copper and cisplatin, while cells expressing Cterminus truncated variant of hCTR1 (CT178) absolutely abolished this ability, suggesting that this motif is crucial for the function of the transporter.

\section{Introduction}

Copper is an essential element for life. The reversible oxidation from $\mathrm{Cu}^{+}$to $\mathrm{Cu}^{2+}$ renders its function as a critical cofactor for a variety of cellular process from oxidative phosphorylation, iron metabolism, free radical detoxification, neurotransmitter synthesis to connective tissue maturation. ${ }^{1,2}$ However, excess copper exhibits toxic effects as a result of generating reactive oxygen species which could damage the cellular components, including protein and DNA. ${ }^{3,4}$ Therefore, the copper homeostasis has to be tightly regulated to avoid toxic effects. All organisms from yeast to humans have developed sophisticated mechanisms by which the cellular accumulation, distribution and detoxification of copper are properly controlled. ${ }^{5,6}$ Malfunctions of copper relevant translation may cause diseases such as neurodegenerative diseases ${ }^{7}$ and two inherited diseases, i.e. Wilson's and Menkes' diseases, which were attributed to copper hyperaccumulation and deficiency respectively. ${ }^{8}$

Although the cellular copper uptake can be carried out by divalent metal transporter 1 (DMT1), ${ }^{9}$ the majority of copper acquisition is mediated by copper transporter 1 (CTR1), ${ }^{10}$ which transports cuprous copper $\left(\mathrm{Cu}^{+}\right)$. Extracellular copper was suggested to exist with an oxidation state of +2 and reduced to $\mathrm{Cu}^{+}$probably by membrane-associated reductases prior to translocation across the cellular membrane via CTR $1 .{ }^{11} \mathrm{CTR} 1$ is a membrane protein with three transmembrane domains, an extracellular $\mathrm{N}$-terminus and an intracellular $\mathrm{C}$-terminus. Three monomers of CTR1 form a channel-like pore, through which $\mathrm{Cu}^{+}$ is transferred across the membrane probably via a series of transchelation reactions between sulfur- (Met and Cys) or nitrogen(His) containing residues located at the inner face of the pore. ${ }^{12-14}$ In addition to CTR1, the low affinity copper transporter CTR2, which mainly located intracellularly with a small fraction on plasma membrane, may account for some of the copper import. ${ }^{15}$

Copper transporter 1 (hCTR1) was also found to modulate the influx of $\mathrm{Pt}(\mathrm{II})$ based anti-cancer drugs such as cisplatin and carboplatin. $^{16,} 17$ Elevated expression levels of human CTR1 (hCTR1) in the human ovarian carcinoma cell were associated with increased cellular uptake of cisplatin, especially at the initial stage. ${ }^{18}$ Similar to copper, cisplatin also rapidly down-regulated hCTR1 in cultured human ovarian carcinoma cells by inducing internalization of the protein from the plasma membrane, which may contribute to the resistance of the drug. ${ }^{19}$ In spite of the importance of CTR1 protein in the uptake and resistance of cisplatin, the molecular details of CTR1-mediated cisplatin transport remain unclear. Considering both $\mathrm{Cu}(\mathrm{I})$ and $\mathrm{Pt}(\mathrm{II})$ are soft acids which shares same preference on the binding to soft ligands including methionine, such a residue on hCTR1 may be important for the transport of both $\mathrm{Cu}^{+}$and cisplatin. Indeed, previous studies indicated that deletion of either the N-terminus of hCTR1 (amino acids 1-45) or the ${ }^{40} \mathrm{MXXXXM}^{45}$ motif eliminated the ability of hCTR 1 to transport copper and partially reduced the capacity to transport cisplatin. ${ }^{20}$ The Met-rich motif $\left({ }^{150} \mathrm{MXXXM}^{154}\right)$ located at the second transmembrane domain (TM2) was also shown to be critical for copper transport. ${ }^{21}$ However, the role of this methionine(Met)-rich motif on cisplatin transport was contradictively reported: Mutations of Met150 and Met154 absolutely abolished the ability of exogenous hCTR1 to stimulate cisplatin uptake in small cell lung cancer cells (SCLC). ${ }^{22}$ On the contrary, cisplatin uptake was significantly enhanced in hCTR1-variant-transfected mouse embryo fibroblasts in which both alleles of CTR 1 had been knocked out $\left(\mathrm{MEF} c t r l^{--}\right){ }^{23}$ Furthermore, the fluorescence resonance energy transfer (FRET) assay of the interactions between yeast CTR1 monomers showed that copper enhanced the FRET in a manner correlated with cellular copper uptake, while cisplatin did not change the FRET. ${ }^{24}$ Mutation of G167, which is located at the third transmembrane domain of hCTR1 and involved in helix packing of the protein, impaired cellular uptake of copper but not cisplatin. $^{22}$ These results indicated that although hCTR1 transports both $\mathrm{Cu}^{+}$and cisplatin, the underlying machanisms 
may differ due to the distinct ligand selectivity, reactivity and structure-dependence between copper and cisplatin.

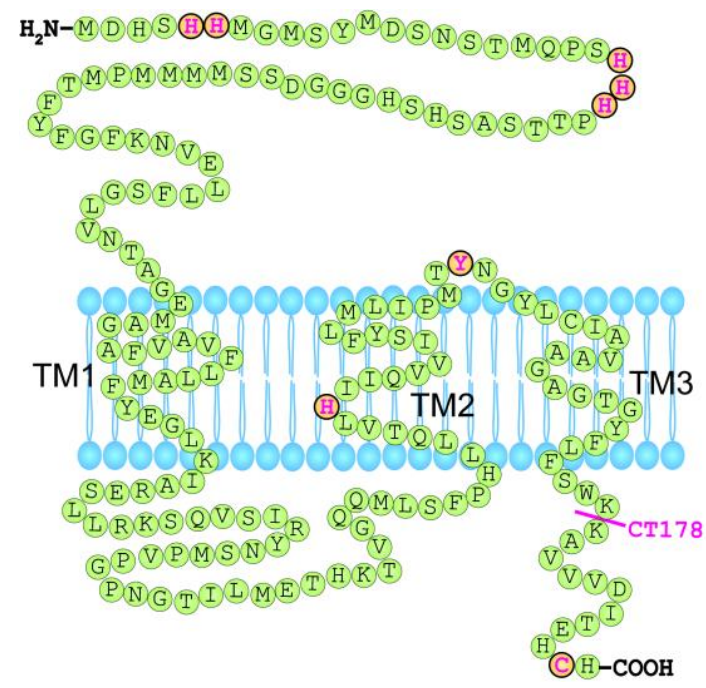

Fig. 1 Schematic diagram showing the topology of hCTR1 The residues or motifs which have been mutated, are highlighted in magenta. All histidine and tyrosine residues were mutated to alanine except H139, which was mutated to arginine, while cysteine was mutated to serine.

Besides methionine residues, other metal-binding amino acids, e.g. histidine and cysteine residues may also play important roles in the uptake of copper and cisplatin by hCTR1. To further investigate the molecular mechanism of hCTR1-meidated transport of these metal compounds, a series of mutant hCTR1 expressing constructs were made and introduced into Hela cells, and their effects on cellular resistances and accumulation of both copper and cisplatin in these stably transfected cells were measured and compared. The role of histidine-rich motifs in the $\mathrm{N}$-terminus was investigated by mutation of histidine residues in H22-24 and H5, 6, 22-24 to alanine (H22-24A and NHA). In one hCTR1 variant, His139 in TM2 was mutated to arginine (H139R) since this residue was proposed to form stacked rings with Met150 and Met154 which provide chelation environment for copper transport. ${ }^{13}$ Two C-terminal variants C189S and CT178 (Deletion of C-terminal fragment ${ }^{179} \mathrm{KAVVVDITEHCH}$ ) were constructed to investigate the role of cysteine residue since it is a soft ligand and suppose to coordiate with both $\mathrm{Cu}$ (I) and $\mathrm{Pt}(\mathrm{II})$ compounds. Tyr156 is located in the putative extracellular loop near TM2. Although not considered as a metal-binding residue, it may play a structrual role in copper entry into the transmembrane pathway. ${ }^{25}$ To further elucidate the role of this residue on hCTR1 function, the Y156A variant was also constructed. All residues and motifs selected for mutation study were highlighted in Fig. 1.

\section{Experimental}

\section{Materials}

Hela cells were purchased from American Type Culture Collection (ATCC, Manassas, VA). Cisplatin, carboplain, transplatin, fetal bovine serum (FBS) and Geneticin (G418) were purchased from Sigma (Sigma-Aldrich) and used withour further process. The platinum drugs were dissolved in OPTI-MEM medium (Invitrogen) to a final concentration of $10 \mathrm{mM}$ prior to usage. Mouse anti-HA primary antibody was obtained from Cell Signaling Technology, Inc. (Danvers, MA). Rabbit anti-CTR1 primary antibody, HRP conjugated anti-mouse and anti-rabbit secondary antibody were purchased from Santa-Cruz Biotechnology, Inc. RIPA lysis buffer was purchased from Pierce Biotechnology. Other chimicals are purchased from either Sigma or USB. All water used are from Millipore.

\section{Construction of wild-type and mutant pcDNA3.1-HA-hCTR1 expression vectors}

The wild-type full length hctrl gene was amplified from the plasmid harboring human $c t r l$ cDNA (kindly provided by Prof. D.J. Thiele ${ }^{26}$ ) by PCR using the forward primer: 5'TTATTAAAGCTTATGGATCATTCCCACCATATG-3' and the reverse primer: 5'-TTATTACTCGAGTTACTAATGGCAATGCTCTGTGATATC-3', by which the Nde I and Xho I restriction sites were introduced at the 5 '- and $3^{\prime}$-ends of the PCR product, respectively. After restriction cleavage, the PCR product was inserted into pcDNA3.1-HA vector (a gift from Prof. Marie C.M. Lin, the University of Hong Kong), which was digested with the same restriction enzymes. All mutant pcDNA3.1-HA-hCTR1 expression vectors were generated using Phusion Site-Directed Mutagenesis Kit (Finnzymes) with primers summarized in Table S1. PCR reactions were performed with Phusion hot star highfidelity DNA polymerase and with plasmid pcDNA3.1-HAhCTR1 as the template (for the NHA mutant, H22-24A was used as a template). All plasmids were confirmed to be correct by DNA sequencing.

\section{Cell Culture}

Hela cells were maintained in Dulbecco's modified Eagle's Medium (DMEM) supplemented with 10\% FBS, $100 \mathrm{U} / \mathrm{ml}$ penicillin $\mathrm{G}, 100 \mu \mathrm{g} / \mathrm{ml}$ streptomycin, and cultured at $37{ }^{\circ} \mathrm{C}, 5 \%$ $\mathrm{CO}_{2}$ incubator.

\section{Plasmid transfection and establishment of stable cells}

One day prior to transfection, $1 \times 10^{6}$ cells in $2 \mathrm{ml}$ DMEM medium were plated so that cells will be $90 \%$ confluent at the time of transfection. To make the transfection complex, the plasmid (2 $\mu \mathrm{g}$ ) was diluted with $100 \mu \mathrm{l}$ OPTI-MEM medium, followed by addition of FuGENE HD transfection reagent $(4 \mu \mathrm{l})$. After incubation at room temperature for 15 minutes, the transfection complex was added to the cells in a drop-wise manner. An enhanced green fluorescent protein (EGFP) expressing plasmid, pcDNA3.1-EGFP (a gift from Prof. Marie C.M. Lin, The University of Hong Kong) was transfected into the nontransfected cells under the same conditions, and the transfection efficiency was evaluated by monitoring the expression of EGFP under fluorescence microscope.

To establish stable cells, cells after 24 hours of transfection were passaged to fresh DMEM/FBS medium containing 0.7 $\mathrm{mg} / \mathrm{ml}$ geneticine (G-418) with the dilution of $1: 8$. Growth medium was renewed every 2-3 days and those dead cells were removed. Cells trasfected with different plasmid were cultured in the presence of G418 for 3-4 weeks until no more dead cells were found and EGFP expression was observed in $>95 \%$ cells 
transfected with EGFP expressing plasmid.

\section{Cell extracts and western blotting}

After washing three times with PBS, the cell pellets were lyzed in 50-100 $\mu$ l RIPA lysis buffer (50 mM Tris- $\mathrm{HCl} \mathrm{pH} 7.4,150 \mathrm{mM}$ $\mathrm{NaCl}, 1 \% \mathrm{NP}-40,0.25 \%$ Na-deoxycholate and $1 \%$ cocktail protease inhibitor) by vortexing and the supernatant was collected by centrifugation $\left(13000 \mathrm{rpm}, 30 \mathrm{~min}, 4{ }^{\circ} \mathrm{C}\right)$. The total protein concentration was determined by $\mathrm{BCA}$ protein assay kit (Novagen). Total protein extracts $(20 \mu \mathrm{g})$ were separated over a $12.5 \%$ tris-glycine gel and electro-blotted onto the PVDF membrane using the Semi-Dry transfer unit Hoefer TE 77 (Amersham). The membrane was blocked with TBS-tween (50 $\mathrm{mM}$ Tris-HCl, $150 \mathrm{mM} \mathrm{NaCl}, 0.1 \%$ Tween-20, $\mathrm{pH}$ 7.5) supplemented with $5 \%$ non-fat milk (blocking buffer) at room temperature for one hour and then incubated with appropriate primary antibody diluted in the blocking buffer at room temperature for one hour. After washed with TBS-tween buffer for three times for 10 mins each, the blot was incubated with corresponding horseradish peroxidase-conjugated secondary antibody with shaking at room temperature for another one hour. The bolt was washed again with TBS-tween buffer three times for 10 mins each. The signal was detected by chemiluminescent ECL system for horseradish peroxidase.

\section{Biotinylation of cell surface proteins}

To label the cell surface proteins, biotinylation of cells was carried out using the thiol-cleavable, cell-impermeable SulfoNHS-SS-biotin reagent (Pierce). Other than stated, all procedures were carried out in ice. Before biotinylation, cells were placed in ice for 15 minutes, the culture medium was removed and the cells were washed once with DMEM containing HEPES buffer (25 $\mathrm{mM}$ ) and three times with PBS containing $1.0 \mathrm{mM} \mathrm{MgCl}_{2}$ and 0.1 $\mathrm{mM} \mathrm{CaCl} 2$. Cells were then incubated with $1.27 \mathrm{mM}$ Sulfo-NHSSS-biotin reagent dissolved in biotinylation buffer $(10 \mathrm{mM}$ triethanolamine, $150 \mathrm{mM} \mathrm{NaCl}, 2 \mathrm{mM} \mathrm{CaCl}_{2}, \mathrm{pH} \mathrm{7.5)}$ for $30 \mathrm{~min}$. Biotinylation was quenched by incubation with glycine $(100 \mathrm{mM}$ dissolved in PBS with $1.0 \mathrm{mM} \mathrm{MgCl}$ and $0.1 \mathrm{mM} \mathrm{CaCl}_{2}$ ) for 30 $\mathrm{min}$, this step was repeated. Cells were lyzed in a lysis buffer (50 $\mathrm{mM}$ Tris- $\mathrm{HCl}, 1 \%$ Triton-X-100, $150 \mathrm{mM} \mathrm{NaCl}$ and $5 \mathrm{mM}$ EDTA, pH 7.5) by vortexing for 10 mins followed by incubation for one hour. The supernatant was collected by centrifugation (13 $\left.000 \mathrm{rpm}, 30 \mathrm{mins}, 4{ }^{\circ} \mathrm{C}\right)$. The supernatant $(10 \%)$ was partially retained for protein concentration determination and the rest was incubated with streptavidin- agarose (Pierce) at the ratio of $100 \mu \mathrm{l}$ agarose per $900 \mu \mathrm{l}$ of supernatant at $4{ }^{\circ} \mathrm{C}$ for overnight. The unbound proteins were removed by centrifugation $(500 \mathrm{rpm}, 5$ mins, $4{ }^{\circ} \mathrm{C}$ ) and the agarose was washed three times with lysis buffer and twice with wash buffer $1(50 \mathrm{mM}$ Tris- $\mathrm{HCl}, 0.1 \%$ Triton-X-100, $150 \mathrm{mM} \mathrm{NaCl}$ and $5 \mathrm{mM}$ EDTA, $\mathrm{pH}$ 7.5) and once with wash buffer $2(50 \mathrm{mM}$ Tris- $\mathrm{HCl}, \mathrm{pH} 7.5)$. Proteins were eluted with $150 \mathrm{mM}$ dithiothreitol (DTT) diluted in SDS-PAGE loading buffer and separated with SDS-PAGE and hCTR1 variant proteins were detected using an anti-HA antibody by western blotting as described above.

\section{Cell survival assay}

Cell proliferation was measured by XTT assay (Roche). Around five thousand cells stably expressing variant hCTR1 proteins in $100 \mu \mathrm{l}$ growth medium were plated into the wells of a 96-well tissue culture plate. After overnight incubation, cells were exposed to the drugs of different concentrations. To measure cell sensitivities towards cisplatin, cells were cultured in $100 \mu \mathrm{l}$ OPTI-MEM medium containing $0-400 \mu \mathrm{M}$ cisplatin for 10 mins. Subsequently, the drug containing cultural medium with the drug was removed and the cells were washed once with pre-warmed PBS and further cultured in $100 \mu \mathrm{l}$ fresh culture medium for another 4-5 days. To measure cell sensitivities towards $\mathrm{CuSO}_{4}$, cells were continually cultured in $100 \mu$ OPTI-MEM medium in the presence of different concentrations of $\mathrm{CuSO}_{4}(0-200 \mu \mathrm{M})$ for 96 hours. And the medium with the metal ion was then removed and the cells were washed once with pre-warmed PBS and further cultured in $100 \mu \mathrm{l}$ fresh culture medium for another 24 hours.

To prepare the XTT labeling mixture (Cell Proliferation Kit II), XTT labeling reagent was mixed with the electron-coupling reagent at 50:1 immediately prior to usage. XTT labeling mixture $(50 \mu \mathrm{l})$ were added to each well and incubated at $37{ }^{\circ} \mathrm{C}$ for $4-24$ hours. The absorbance at $495 \mathrm{~nm}$ was measured with a reference wavelength at $695 \mathrm{~nm}$. The survival curve was generated by plotting the absorbance $\left(\mathrm{A}_{495}-\mathrm{A}_{695}\right)$ versus the metal concentrations.

\section{Cellular metal ions accumulation}

Cells stably expressing variant hCTR1 proteins were seeded in 6well plates one day prior to the expreiment. After 24 hours, cells were exposed to either $\mathrm{CuSO}_{4}(100 \mu \mathrm{M})$ for 1 hour or platinumcontaining agents (cisplatin, transplatin or carboplatin, $100 \mu \mathrm{M}$ ) for $10 \mathrm{mins}$ by the addition of metal/drug containing OPTI-MEM medium. The medium was removed after incubation and cells were washed three times with ice-cold PBS. Cells were colleted and washed with ice-cold PBS once. For each sample, half of the cells were lyzed using RIPA buffer and the total protein concentration was measured by BCA protein assay kit. The remaining cells were digested with $65 \%$ nitric acid $(50 \mu \mathrm{l})$ at 65 ${ }^{\circ} \mathrm{C}$ for overnight to thoroughly dissolve all cellular debris. The mixture was diluted in the following day with buffer containing $0.1 \%$ Triton X-100, $1.4 \%$ nitric acid and $2 \mathrm{ppb}$ Tl. Metal concentrations were determined by inductively coupled plasma mass spectroscopy (ICP-MS, 7500a Agilent technologies) and were normalized with total protein concentration.

\section{Results and discussion}

\section{HA-hCTR1 Expression in Transfected Cells}

The effects of the wild-type and hCTR1 mutants on cellular uptake and toxicity of copper and platinum-containing drugs were investigated using cisplatin-sensitive Hela cell line. ${ }^{27}$ A series of stably transfected Hela cell lines harboring the wild-type or mutant hCTR1 including H22-24A, NHA, H139R, Y156A, C189S and CT178 were established. An HA-tag was added to the $\mathrm{N}$-termini of these proteins to facilitate the detection of proteins by Western blot. The expression of exogenous hCTR1 in the stably transfected cells was confirmed by western blot using 
antibodies against the HA-tag and the internal region of human CTR1 (Fig. 2). A band migrating at $\sim 40 \mathrm{kDa}$ was detected by both anti-HA and anti-CTR1 antibodies corresponding to hCTR1 expressed from the transfected plasmid. This is in agreement with previous reports ${ }^{28,29}$ that hCTR1 was $\mathrm{N}$ - and O-glycosylated at Asn-15 and Thr-27 respectively, with a molecular weight of ca. $37 \mathrm{kDa}$ for the mature protein. Therefore, the exogenous HAhCTR1 expressed from the introduced plasmids was successfully glycosylated.

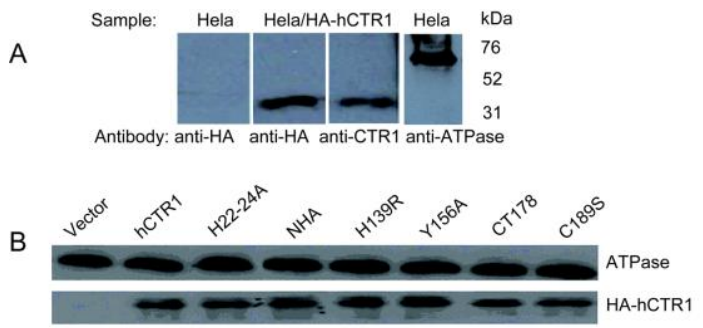

Fig. 2 Western blot analysis of exo-hCTR1 expressed in total cell lysates (A) and cell surface (B) The antibody against the $\beta$-subunit of $\mathrm{Na}^{+}, \mathrm{K}^{+}$-ATPase was used as the loading control and marker for membrane fraction.

As shown in Fig. 2B, the successful biotinylation of hCTR1 proteins indicated that both the wild-type and different mutants of hCTR1 were correctly localized on the plasma membrane, as was the $\beta$-subunit of $\mathrm{Na}^{+} / \mathrm{K}^{+}$-ATPase, a plasma membrane marker. The expression levels of hCTR1 variants were comparable to the wild-type protein and also migrated at $\sim 40 \mathrm{kDa}$, corresponding to the HA-tagged monomer as observed previously. ${ }^{30,31}$ Based on these studies, we found that all the residues or motifs examined in this study are not critical determinants for the correct cellular distribution of hCTR1.

\section{hCTR1 Regulation of Copper Uptake and Cytotoxicity}

The basal copper level was tested by incubation of cells transfected with hCTR1 variant plasmids in DMEM medium in the presence of ca. $0.3 \mu \mathrm{M}$ copper ions. The basal copper level in hCTR1-transfected cells was found to be 2.4-fold higher than that in the empty vector transfected cells as shown in Fig. 3A, in agreement with previous studies that over-expression of hCTR1 in mammalian cells stimulated copper uptake. ${ }^{21,} 32$ Hela cells expressing hCTR1 variants of H139R reduced basal copper levels by $22 \%$ with regards to that in the wild-type protein expressed cells (Hela/hCTR1). Deletion of the C-terminal fragment ${ }^{179}$ KAVVVDITEHCH exhibits similar effects to the C189S and Y156A variant in terms of copper uptake, which reduced basal copper levels by $\sim 60 \%$. Unexpectedly, the basal copper accumulation levels in the cells expressing the N-terminal variants increased by 1.2-fold and 1.5-fold for H22-24A and NHA respectively in comparison with that in the wild-type hCTR1 expressed cells (Fig. 3A).

To measure the rates of copper accumulation, cells transfected with variant hCTR1 expressing vectors were exposed to $100 \mu \mathrm{M}$ $\mathrm{CuSO}_{4}$ for $1 \mathrm{~h}$. Similar to basal copper accumulation experiment, the cells expressing the wild-type hCTR1 enhanced copper uptake by 2-fold compared with the empty vector transfected cells (Fig. 3B). The cells expressing the H139R and Y156A variants reduced the rates of copper accumulation by $34 \%$ and $39 \%$, respectively. Deletion of the 12 amino acids in the $\mathrm{C}$ terminus $\left({ }^{179} \mathrm{KAVVVDITEHCH}\right)$ disrupted copper transport, whereas the single mutation of Cys 189 retained $83 \%$ capacity of copper transport. However, the copper accumulation in the H2224A and NHA expressing cells are only slightly higher than that in the wild-type protein transfected cells.

To examine the expression of hCTR1 variants on the cellular sensitivity towards copper toxicity, the $\mathrm{IC}_{50}$ values of $\mathrm{CuSO}_{4}$ in these transfected cells were further measured by the XTT method. Cells were exposed to increasing concentrations of $\mathrm{CuSO}_{4}$ for 96 hrs prior to measurement. The cells expressing the wild-type hCTR1 was 4.3-fold more sensitive to copper than those cells transfected with an empty vector $\left(\mathrm{IC}_{50} 16.1 \mu \mathrm{M}\right.$ versus $69.4 \mu \mathrm{M}$, Fig. 3C and 3D). Cells expressing the H22-24A and NHA showed slightly more sensitive to copper than the wild-type hCTR1 expressed cells, with $\mathrm{IC}_{50}$ of 14.3 and $10.6 \mu \mathrm{M}$, respectively. Mutations of either His-139, Tyr-156 and Cys-189 reduced the cell sensitivities towards copper, indicating that these residues in hCTR1 are important for copper transport. Among all hCTR1 variants, truncation of C-terminus (CT178) rendered the transfected cells the most insensitive for copper accumulation, in agreement with our pervious results that the deletion of the C-terminal disabled copper uptake. Theses results clearly demonstrated that higher copper accumulation in hCTR1 variant transfected cells would lead to higher cytotoxicity.
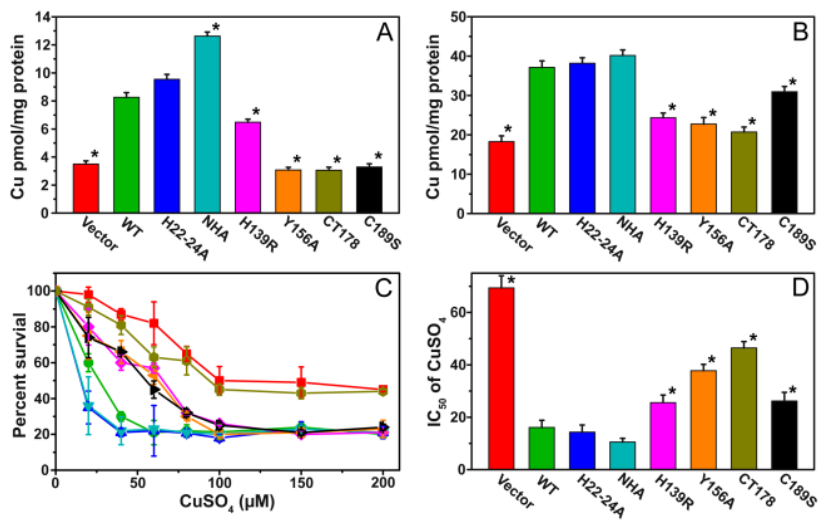

Fig. 3 Effects of mutations of hCTR1 on copper uptake and sensitivity towards the cytotoxicity of copper in Hela cells (A) Total basal copper accumulation in cells cultured in DMEM. (B) Total copper accumulation upon exposure to $100 \mu \mathrm{M} \mathrm{CuSO}_{4}$ for 1 hour. (C) Survival rates of Hela cells harboring empty vector $(\square)$; the wild-type hCTR1 (๑); H22-24A $(\Delta)$; NHA $(\nabla)$; H139R $(\diamond)$; Y156A $(\triangleleft)$; C189S $(\bullet)$ and CT178 (), after $96 \mathrm{~h}$ continuous exposure to increasing concentrations of $\mathrm{CuSO}_{4}(20 \mu \mathrm{M}-200 \mu \mathrm{M})$. (D) $\mathrm{IC}_{50}$ of $\mathrm{CuSO}_{4}$ with Sigmoidal fitting. Error bars represent standard derivations from four independent experiments. The asterisk $\left(^{*}\right)$ indicates $p<0.05$ as determined by the Student's $t$ test, compared with the wild-type hCTR1.

Based on the result above, it seems the His-139, Tyr-156 and Cys-189 residues were partially involved in the transport of $\mathrm{Cu}^{+}$ ions, while the intact C-terminus is crucial for the normal function of hCTR1 on copper transport. However, the role of two His-rich motifs $\left({ }^{3} \mathrm{HXHH}\right.$ and $\left.{ }^{22} \mathrm{HHH}\right)$ at the N-terminus of hCTR1 in uptake of copper of basal and excess level seems controversial. Hela cells expressing variants of H22-24A and NHA, especially NHA, exhibited obviously higher basal copper 
accumulation compared with cells expressing the wild-type hCTR1, while no such effect was observed when extra copper ions $(100 \mu \mathrm{M})$ were added to the cultural medium. It was believed that the extracellular free or protein bound $\mathrm{Cu}^{2+}$ ions were reduced by unidentified reductases or reducing agents (e.g. ascorbate) at the cell surface to $\mathrm{Cu}^{+}$prior to being transferred across the membrane via hCTR1. Our preliminary study showed that $\mathrm{Cu}^{2+}$ bound to hCTR1 via the two His-rich motifs with moderate affinity $\left(K_{d}=9.8 \mu \mathrm{M}\right)$ (unpublished data). It is likely that at lower copper concentrations (DMEM contains $\sim 0.3 \mu \mathrm{M}$ $\mathrm{Cu}$ ), binding of $\mathrm{Cu}^{2+}$ to the His-rich motifs might inhibit the reduction of $\mathrm{Cu}^{2+}$ to $\mathrm{Cu}^{+}$and subsequently reduce the accumulation of copper in cells. Under copper replete conditions, the excess copper would result in the system less sensitive towards the effects of the His-rich motifs.

\section{Effects of hCTR1 on Initial Influx of Platinum-based Drugs}

The effects of hCTR 1 on the influx of platinum containing drugs were examined similarly by comparing the cellular platinum accumulation between Hela and Hela/hCTR1 cell lines. Since the major contribution of hCTR1 to cisplatin uptake occurred in the initial phase of cisplatin influx ${ }^{33}$ here we determined the initial influx of platinum drugs by exposing cells to cisplatin, carboplatin or transplatin at the concentration of $100 \mu \mathrm{M}$ for 10 mins. The cell associated platinum contents were measured by ICP-MS and the results were summarized in Fig. 4. Hela cells expressing exogenous hCTR1 resulted in a 2.2-fold increase in platinum accumulation compared with cells transfected with an empty vector under identical conditions. Such effects are even more pronounced for carboplatin, i.e. a 2.9-fold increase of platinum contents in Hela cell was observed due to the expression of exogenous hCTR1. In contrast, the platinum contents were almost the same for the two types of cells when exposed to transplatin, indicating that hCTR1 did not stimulate the cellular accumulation of transplatin. The initial influx of transplatin in Hela/hCTR1 cells is 1.1-fold higher than that of cisplatin, while the initial influx of carboplatin is $58 \%$ of that of cisplatin. Our results demonstrated that transplatin entered cells via hCTR1independent pathway, in agreement with a previous report that reexpression of hCTR 1 in mouse embryo fibroblasts $\mathrm{ctrl}^{-/}$cells did not stimulate the cellular accumulation of transplatin. ${ }^{23}$
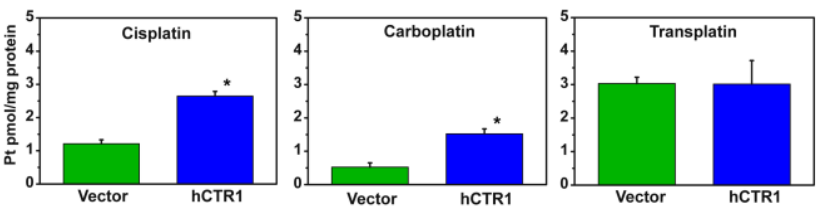

Fig. 4 Pt-associated with Hela cells transfected with an empty vector or hCTR1 expression vector, error bars represent standard derivations from six separate cultures. The asterisk indicates $p<0.05$ as determined by the Student's $t$ test.

\section{hCTR1 Regulation of Cisplatin Uptake and Cytotoxicity}

The effects of the expression of hCTR1 variant proteins on the initial influx of cisplatin in Hela cells were examined by exposing cells to $100 \mu \mathrm{M}$ cisplatin for $10 \mathrm{~min}$ and the cell associated platinum contents were measured. As shown in Fig. 5A, C189Stransfected cells significanly inhibited platinum uptake with $42 \%$ of the incremental increase in platinum content produced by the wild-type hCTR1. Notably, C-terminal truncation (CT178) impaired the cisplatin transport function of hCTR1 (Fig. 5A), whereas the platinum content increased by 1.1-fold compared with that for the empty vector transfected cell, or $7.3 \%$ of the incremental increase in that produced by the wild-type hCTR1. However, it was found that cells expressing either of hCTR1 variants of H22-24A, NHA, H139R and Y156A preserved cisplatin transport function of hCTR1.

The effects of 10 mins exposure to increasing amounts of cisplatin on the growth rate of Hela cells transfected with hCTR1 variant plasmids during a subsequent period of 4 days were investigated (Fig. 5B). As expected, the expression of the wildtype hCTR1 increased the sensitivity of Hela cells to cisplatin by a factor of 2.1-fold, with the $\mathrm{IC}_{50}$ decreased from 193.1 \pm 9.6 to $92.4 \pm 5.9 \mu \mathrm{M}$ (Fig. 5C). Consistent with their abilities to enhance cisplatin uptake to a similar extent to the wild-type protein, expression of either H22-24A, NHA, H139R or Y156A variants increased cisplatin cytotoxicity by 1.9-, 2.1-, 1.7- and 2.1-folds, with $\mathrm{IC}_{50}$ of $102.9 \pm 12.1,90.4 \pm 5.6,115.2 \pm 5.2$ and $93.9 \pm 4.1 \mu \mathrm{M}$, respectively. However, expression of either C189S or CT178 rendered Hela cells less sensitive to platinum compared with that of the wild-type hCTR1, with the $\mathrm{IC}_{50}$ values of $131.2 \pm 20.1$ and $165.8 \pm 9.3 \mu \mathrm{M}$, respectively. Significantly, the correlation between cisplatin accumulation and cytotoxicity of the drug among hCTR1 variants expressed cells was noted, demonstrating that the drug accumulated by the exogenous hCTR1 was successfully transferred to the critical targets such as DNA.
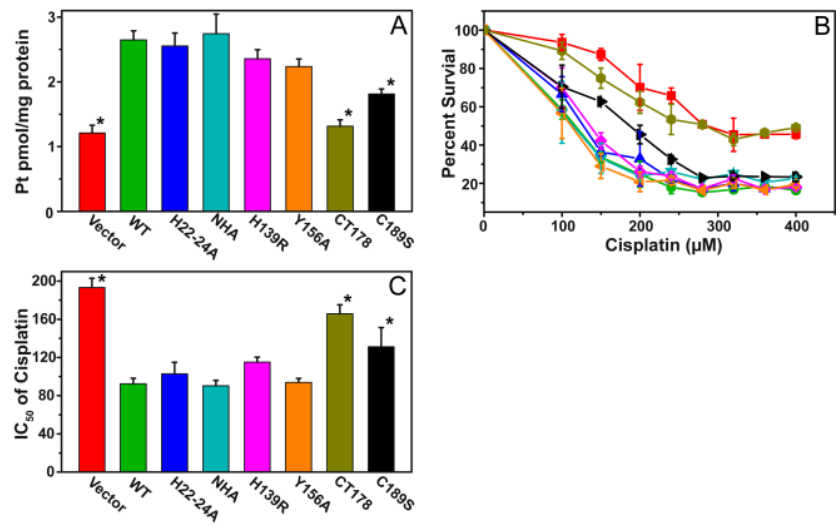

Fig. 5 Effects of mutations of hCTR1 on cisplatin accumulation and sensitivity towards the cytotoxicity of cisplatin in Hela cells (A) Total platinum accumulation upon exposure to $100 \mu \mathrm{M}$ cisplatin for 10 mins. (B) Survival rates of Hela cells harboring empty vector $(\square)$; the wild-type

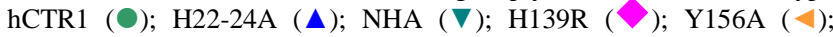
C189S $(\triangleright)$ and CT178 ( ), after exposure to increasing concentrations of cisplatin for 10 mins and then cultured in normal media for $96 \mathrm{hrs}$. (C) $\mathrm{IC}_{50}$ of cisplatin determined with Sigmoidal fitting. The error bars represent standard derivations from four independent experiments. The asterisk indicates $p<0.05$ as determined by the Student's $t$ test, compared with the wild-type hCTR1.

Like in the case of copper, among all the variant forms of hCTR1 examined, only truncation at the C-terminus (CT178) completely abolished hCTR 1 mediated cellular uptake of cisplatin, whereas the single mutation at Cys189 only partially reduced the cisplatin uptake. Therefore, the C-terminus of hCTR1 is required for both the cellular uptake of copper and cisplatin. In 
the absence of Cys189, the adjacent two His residues (H188 and $\mathrm{H} 190)$ might serve as the coordination ligands for $\mathrm{Cu}(\mathrm{I})$ and probably $\mathrm{Pt}(\mathrm{II})$, as supported by the observation of $\mathrm{Cu}-\mathrm{N}$ (His) coordination signal in the EXAFS spectra of hCTR1 when incubated with $\mathrm{Cu}^{+} \cdot{ }^{13}$ According to our previous studies, cisplatin was presumed to be transported via hCTR1 in its monofunctional form $\left(\right.$ cis- $\left.\left[\mathrm{PtCl}\left(\mathrm{NH}_{3}\right)_{2}\right]^{+}\right){ }^{34}$ Thus, similar to $\mathrm{Cu}^{+}$, the monofunctional cisplatin is likely to pass through the pore created by three monomers of hCTR 1 via a series of trans-chelation reactions between Met154 and Met150. ${ }^{13}$ The C-terminal Cys189 (or ${ }^{188} \mathrm{HCH}$ motif) may participate in the sequential transchelation reactions, accepting copper and cisplatin from the upper ring probably formed by three Met150 and then handling them down.

The requirement of Met-rich motifs at the N-terminus of hCTR1 for cisplatin transport has been extensively studied, ${ }^{20}$ however, the role of its His-rich motifs $\left({ }^{3} \mathrm{HXHH}\right.$ and $\left.{ }^{22} \mathrm{HHH}\right)$ was poorly understood. We found mutations at the His-rich motifs did not affect the ability of hCTR1 to stimulate the cellular uptake of cisplatin, suggesting that these residues play a less important role in cisplatin transport. This is supported by the recent study showing that cisplatin binds predominately to the $\mathrm{N}$ terminus methionine residues but not histidine residues of hCTR $1 .^{34,}{ }^{35}$ Recent electro-crystallographic analysis demonstrated that the second transmembrane helix of hCTR1 serves as the key element lining the pore created by three monomers of hCTR $1 .{ }^{13}$ Along the pore, Met154, Met150 and H139 were suggested to form three stacked rings which mediated copper influx through a series of trans-chelation reactions. Previously, intensive studies have demonstrated the importance of these three residues in hCTR1-mediated copper transport. ${ }^{20-22,}$

${ }^{25}$ We show here that the mutation of $\mathrm{H} 139$ to arginine decreased copper but not cisplatin accumulation in Hela cells, indicating that H139 of hCTR1 play little role in cisplatin transport, in agreement with a previous report that mutation of H139 to alanine (H139A) did not significantly affect its ability to transport cisplatin in $\mathrm{Ctr}^{-/-}$MEF system. ${ }^{23}$ Similarly, Y156 located at the second transmembrane segment plays an important role in the uptake of copper but not cisplatin in Hela cells. Previously, it was also observed an $80 \%$ reduction at the copper transport rate compared with that of the wild-type protein in Sf9 cells expressing the Y156A variant. ${ }^{25}$ This residue is located closely to Met154 and may structurally help to place Met154 appropriately. The discrepancy occurred for the these variants such in mediating copper and cisplatin uptake, possibly attributed to variation in the local structure or charge for these variant proteins.

\section{Conclusion}

The effect of hCTR1 mutations on cellular accumulation and sensitivity enhancement of cytotoxicity of both copper and $\mathrm{Pt}$ drugs was investigated. The necessity of the C-terminus of hCTR1 in cisplatin uptake was addressed in this report, which might shed light on the mechanism of cisplatin uptake. Our combined data suggested that despite of both copper and cisplatin passing through the trimeric pore of hCTR1 via a series of transchelation reactions, the detailed molecular mechanism might be different. Further biological and structural investigations of the transport are therefore warranted.

\section{Acknowledgement}

This work was supported by RGC of Hong Kong (703808, HKU1/07C, 704909, 705310 and N_HKU75209), Croucher Foundation and the University of Hong Kong. We thank D.J. Thiele (University of Michigan) for the hCTRl gene, and Marie C.M. Lin (University of Hong Kong) for pcDNA3.1-HA and pcDNA3.1-EGFP vectors, Z.J. Guo (Nanjing University) for helpful discussion.

\footnotetext{
${ }^{a}$ Department of Chemistry, The University of Hong Kong, Hong Kong, China. Fax: (+852) 2857 1586; Tel: (+852) 2859 8974; E-mail: hsun@hku.hk

${ }^{b}$ School of Life Sciences, Shenzhen University, Shenzhen, China. $\dagger$ Electronic Supplementary Information (ESI) available: [Primers used for generation of pcDNA3.1-HA-hCTR1 variants are listed in Table S1]. See DOI: $10.1039 / \mathrm{b} 000000 \mathrm{x} /$
}

1. D. W. Cox, Br. Med. Bull., 1999, 55, 544-555.

2. C. D. Vulpe and S. Packman, Annu. Rev. Nutr., 1995, 15, 293-322.

3. I. Fridovich, Science, 1978, 201, 875-880.

4. B. Halliwell, Am. J. Med., 1991, 91, 14S-22S.

5. M. C. Linder, N. A. Lomeli, S. Donley, F. Mehrbod, P. Cerveza, S. Cotton and L. Wotten, Adv. Exp. Med. Biol., 1999, 448, 1-16.

6. M. C. Linder, L. Wooten, P. Cerveza, S. Cotton, R. Shulze and N. Lomeli, Am. J. Clin. Nutr., 1998, 67, 965S-971S.

7. D. J. Waggoner, T. B. Bartnikas and J. D. Gitlin, Neurobiol. Dis., $1999,6,221-230$.

8. P. C. Bull and D. W. Cox, Trends Genet., 1994, 10, 246-252.

9. M. Arredondo, P. Munoz, C. V. Mura and M. T. Nunez, Am. J. Physiol. Cell Physiol., 2003, 284, C1525-1530.

10. P. V. van den Berghe and L. W. Klomp, J. Biol. Inorg. Chem., 2010, 15, 37-46.

11. R. Hassett and D. J. Kosman, J. Biol. Chem., 1995, 270, 128-134.

12. S. G. Aller and V. M. Unger, Proc. Natl. Acad. Sci. USA, 2006, 103, 3627-3632.

13. C. J. De Feo, S. G. Aller, G. S. Siluvai, N. J. Blackburn and V. M. Unger, Proc. Natl. Acad. Sci. USA, 2009, 106, 4237-4242.

14. S. B. Howell, R. Safaei, C. A. Larson and M. J. Sailor, Mol. Pharmacol., 2010, 77, 887-894.

15. J. Bertinato, E. Swist, L. J. Plouffe, S. P. Brooks and R. L'Abbe M, Biochem. J., 2008, 409, 731-740.

16. A. K. Holzer, G. H. Manorek and S. B. Howell, Mol. Pharmacol., 2006, 70, 1390-1394.

17. X. Lin, T. Okuda, A. Holzer and S. B. Howell, Mol. Pharmacol., 2002, 62, 1154-1159.

18. A. K. Holzer, G. Samimi, K. Katano, W. Naerdemann, X. Lin, R. Safaei and S. B. Howell, Mol. Pharmacol., 2004, 66, 817-823.

19. A. K. Holzer, K. Katano, L. W. Klomp and S. B. Howell, Clin. Cancer Res., 2004, 10, 6744-6749.

20. C. A. Larson, P. L. Adams, D. D. Jandial, B. G. Blair, R. Safaei and S. B. Howell, Biochem. Pharmacol., 2010, 80, 448-454.

21. S. Puig, J. Lee, M. Lau and D. J. Thiele, J. Biol. Chem., 2002, 277 26021-26030.

22. Z. D. Liang, D. Stockton, N. Savaraj and M. Tien Kuo, Mol. Pharmacol., 2009, 76, 843-853.

23. C. A. Larson, P. L. Adams, B. G. Blair, R. Safaei and S. B. Howell, Mol. Pharmacol., 2010, 78, 333-339.

24. D. Sinani, D. J. Adle, H. Kim and J. Lee, J. Biol. Chem., 2007, 282 , 26775-26785.

25. J. F. Eisses and J. H. Kaplan, J. Biol. Chem., 2005, 280, 3715937168

26. J. Lee, M. M. Pena, Y. Nose and D. J. Thiele, J. Biol. Chem., 2002, 277, 4380-4387. 
27. J. Zisowsky, S. Koegel, S. Leyers, K. Devarakonda, M. U. Kassack, M. Osmak and U. Jaehde, Biochem. Pharmacol., 2007, 73, 298-307.

28. A. E. Klomp, B. B. Tops, I. E. Van Denberg, R. Berger and L. W. Klomp, Biochem. J., 2002, 364, 497-505.

29. E. B. Maryon, S. A. Molloy and J. H. Kaplan, J. Biol. Chem., 2007, 282, 20376-20387.

30. J. F. Eisses and J. H. Kaplan, J. Biol. Chem., 2002, 277, 2916229171.

31. Y. Guo, K. Smith, J. Lee, D. J. Thiele and M. J. Petris, J. Biol. Chem., 2004, 279, 17428-17433.

32. I. S. Song, N. Savaraj, Z. H. Siddik, P. M. Liu, Y. J. Wei, C. J. Wu and M. T. Kuo, Mol. Cancer. Ther., 2004, 3, 1543-1549.

33. C. A. Larson, B. G. Blair, R. Safaei and S. B. Howell, Mol. Pharmacol., 2009, 75, 324-330.

34. X. Wang, X. Du, H. Li, D. S. Chan and H. Sun, Angew. Chem. Int. Ed. Engl., 2011, 50, 2706-2711.

35. Z. Wu, Q. Liu, X. Liang, X. Yang, N. Wang, X. Wang, H. Sun, Y. Lu and Z. Guo, J. Biol. Inorg. Chem., 2009, 14, 1313-1323. 